\title{
Research Progress of Genetic Immunization and Confronting Problems
}

\author{
Zhang Dandan ${ }^{1}$, Gao Chengguang ${ }^{2, *}$ \\ ${ }^{1}$ College of Life Sciences, Baicheng Normal University \\ ${ }^{2}$ College of Life Sciences, Baicheng Normal University
}

Keywords: Genetic immunization; Research; Problem

\begin{abstract}
: as an important discovery in the history of human medicine, genetic immunization makes important contribution to the development of medical science and human's healthy life. The progress of research on genetic immunization, which needs us to pay close attention to, is about the fate of all mankind. And the problem existing in the research of genetic immunization also needs us to focus on and propose corresponding countermeasures from different perspectives so as to safeguard human life and health and maintain the progress of medical technology.
\end{abstract}

\section{Birth and development process of gene vaccine}

Gene vaccine is the third generation vaccine following pathogen vaccine and subunit vaccine, and it was called the third vaccine revolution by some people. The advent of gene vaccines provides more methods and possibilities for human to cure diseases, becoming a milepost across human medical technology.

\subsection{First generation vaccine}

Emergence of the first generation vaccine was mainly represented by Janner's vaccination in human body with against smallpox and attenuated rabies vaccine developed by Pasetur. Using the first generation vaccine is mainly to extract completed pathogen and then inoculate to human body under nontoxic or attenuated conditions to cause immune reaction by pathogen and human contact, and thus help human avoid dangers of many diseases. The first generation vaccine is mainly for smallpox, measles and animal infectious diseases. The advent of the first generation vaccine provides a new approach for human to resist all kinds of diseases, becoming an important node in the history of human medicine.

\subsection{Second generation vaccine}

Emergence of the second generation vaccine mainly uses genetic engineering technology to separate corresponding genes having antigen protein with strong immunogenicity and then generate corresponding antigen as vaccine by protein recombination. It also can manually connect antigen segment from sources of different pathogens to form subunit vaccine. A typical example is Hepacare.

\subsection{Gene vaccine}

Gene vaccine refers to the eukaryotic expression vector constructed by extending antigen gene fragment on the basis of coding the gene fragment with specific antigen and then connecting carriers. Gene vaccine is an improvement based on the first and the second generation vaccines, which can not only form antibody like the first generation vaccine by inoculating live pathogen but also amplify the chosen gene fragment like the second generation vaccine.

Comparing to the first and the second generation vaccines, gene vaccine has more advantages. Firstly, as for practicability, the equipment used to produce gene vaccine is simpler, and the vaccine can be stored longer and therefore convenient for storage and transportation, which avoids the awkward situation in backward areas of being short of gene vaccine due to traffic inconvenience.

Secondly, gene vaccine is more applicable. For instance, gene vaccine has achieved good results in researches of many fields like virus, parasite, etc. As it were, gene vaccine has very broad 
development prospect. With further studies in future, gene vaccine may more helpful for dealing with tumor and cancer.

Additionally, plasmid can be stored within human body for a relative long time, and it can generate pathogenic antibody continuously to provide limitless elements needed to combat viruses so as to keep human body away from viral invasion after vaccination.

Finally, use of gene vaccine can also avoid problem of tolerance. After entering the human body, gene vaccine can move constantly and stimulate the immune balance of human body, thus make our body more tolerant.

This proves that, human's technology is always in constant change and development, and there is no doubt that the gene vaccine newly developed has better using effect and broader application prospect. Therefore, constant research on the basis of genetic immunization may let science serve the society better.

\section{Step analysis of genetic immunization}

\subsection{Analysis on target gene}

First of all, we shall make clear before research of genetic immunization that whether it can be expressed in mammalian cell, whether there is rare codon or intron, and whether can correct shearing be conducted in mammalian cell.

\subsection{Selection of carrier}

Generally, the carrier of genetic immunization is plasmid. It is because that plasmid's own characteristics give genetic immunization better carrying capacity to maintain production even during reproduction of Escherichia coli, but it won't reproduce in mammalian cells. Therefore, it can achieve good gene immunization effect while minimizing the impact of interference and other factors.

\subsection{Confirmation of coding sequence by sequencing}

When target gene is obtained via PCR cloning, the occurrence of mutation, especially nonsense mutation, shall be avoided since Taq enzyme is lack of the correction action of $3^{\prime}-5$ ' externally-tangent and thus there is certain Mismatch Rate.

\subsection{Performing in vitro transcription / translation experiment with rabbit reticulocyte lysate}

Through this experiment, translation synthesis of target protein in mammalian cell can be guaranteed; and then, by conducting transient transfection experiment with mammalian cell line including $\mathrm{CHO}$, COS, HePG2, etc, it is ensured that carrier can correctly guide to target protein synthesis in mammalian cell; and method for examination including immunofluorescence, immunoblotting or immunoprecipitation.

\section{Recent Progress in gene immunization research}

\section{1 mRNA gene immunization}

Since the stability of RNA is not high in human body, it is not as stable as DNA that can transfer RNA by combining with different tissues and organs. Therefore, RNA's function is limited in human body. However, from the results of various tests and the theoretical perspective, the effect of RNA vaccine is better than that of DNA. It is mainly because that RNA may not directly combine with DNA. So, the use of RNA in vaccine may reduce the possibility of various mutations. Currently, some people have developed the vaccine of RNA, and use method is that the injection of RNA vaccine can continuously induce antibodies within mammalian body and let the titer of antibody reach a higher level. 


\subsection{Expression library immunization}

Though gene immunization has broad development prospect, there is a problem exists in its carrier: it has no way to make clear which gene shall be included in the carrier of gene immunization. This problem has always perplexing scientists, especially when encountering with pathogen with relatively large genome, the problem of carrier in gene immunization is even more prominent. To solve this problem, scientists developed expression library immunization. The method of such immunization is to digest and cut genome DNA firstly to make DNA into gene with protective property, and then connect digestion fragment to eukaryotic expression vector respectively; conduct immunization on many animals with all or part of gene base so as to understand which part of expressed proteins of genome or disintegrated pathogen can produce effective immune protection. ELI is a good gene vaccine screening tool which is able to discover suitable vaccines systematically and objectively.

\subsection{Mucosal gene immunization}

Mucosal gene immunization plays a vanguard resistance role during the resistance process of mucosal infection, so it can avoid spread of pathogens in human body to cause diseases. The using objects of mucosal gene are mainly sexually transmitted diseases. In recent years, sexually transmitted diseases are on the rise. Because of the impact of culture and the opening of sexual concepts, the spread of these diseases have a higher probability of transmission. Mucosal gene immunization can deal with such diseases spread well. It can exclude the pathogen from first pass and thus reduce the probability of sexually transmitted diseases. As it should be, mucosal gene immunization is not just be used on sexually transmitted disease. It accounts for an important proportion in the practical application and operation of sexually transmitted disease.

\subsection{Internet interconnection of gene immunization}

Research of gene immunization needs a lot of manpower and material resources, but fighting against disease and protecting human health is a common topic in the world. For a long time, many countries invested a lot of time and manpower as well as financial resources into the research of gene immunization. And with the development of internet information technology and the deepening of cross border medical collaboration, a site specializing in gene immunization research was built on internet in 1995, which let all scientists dedicated in the research of gene immunization in the world use existing literature and research results as almost all known research data on gene immunization can be found on this site, and there are a lot of related questions about the gene immunization, such as special lecture on gene immunization and job-hunting information. It is a site with rich content and connecting all experts and scholars of gene immunization in the world to make efforts and contributions for gene immunization research.

\section{Confronting problems of gene immunization research}

\subsection{Pathogen's characteristics of high explosion and diversification}

The update of gene immunization technology has always been synchronized. The basic research thinking is to study based on the existing disease symptoms and those can be predicted in future, and take this as research direction to develop corresponding gene immunization results. However, in modern society, problems like destroyed environment, aggravated industrial pollution, a large number of food additives, etc make people's body function more fragile than before, and various new diseases become threats to human health and safety. Pathogen's characteristics of high explosion and diversification broaden the research direction of gene immunization, but these also weaken researchers' energy for it is difficult for them to have in-depth research in a certain field.

\subsection{Human tolerance}

As mentioned above, gene immunization is safer and more efficient than the previous vaccines. Meanwhile, gene immunization can constantly stimulate and break the original homeostatic balance 
and let gene immunization always be of high tolerance, and thus ensure better and more durable effect of gene immunization.

However, the ecological environment in modern human body may have changed, so there shall be more development for gene immunization. From a very common point of view, although gene immunization may stimulate human body to form tolerance, it won't continue to change the original environment of human body. It can't be denied that gene immunization will change human environment more as time goes by even under constant stimulation. Even if gene immunization can meet the current treatment requirements, further research is still needed to adapt to the changing needs of human body.

\section{Solution for the research problems of gene immunization}

It can be seen from the direction of this article that technology update of immune research can't be avoided and it is also the need of development based on reality. It is realizable to technically improve it so as to promote the solution of technical problems. Therefore, this paper emphasizes the divergent thinking from the aspects including society, system, education, etc.

\subsection{Promoting medical innovative education}

Both genetic immunity and other studies need professionals to work on it. Education is the important base for cultivating such professionals and the key point to ensure the follow up development of gene immunization. Meanwhile, it is also the key point to determine the future direction and innovation point of gene immunization, and thus we need to actively promote the innovative medical education.

In the existing medical education system, more research laboratories must be built to invest better equipment and more funds to stimulate the influx of talent and let them conduct innovation works under advanced equipment and conditions. Additionally, cooperation between school education and hospital units shall also be strengthened. In case of new cases appear in the hospital, research laboratories shall be informed to let researchers study on and then feedback the results to clinical treatment, and thus shorten the time from research to practice.

\subsection{Strengthen system and mechanism improvement}

For a long time, China is not an innovative country. Initiated by government, China has been made great progress in recent years. But the institutional mechanism of innovation research still needs further improvement. Firstly, welfare of researchers in institutional mechanism must be improved. After making clear of everyone's position and post responsibility, different standards of treatment may be formulated as per post responsibility; researchers having major research achievements can get rewards so as to boost the motility of researchers in gene immunization research and then conduct their own works without the worries behind. Secondly, exchanges and communications between researches on gene immunization in China and advanced studies abroad must be improved from the perspective of institutional mechanism; corresponding mechanism can be established to promote the discussion and sharing on gene immunization research between both sides, commonly promote the development of gene immunization research, and ensure further development of gene immunization research by official institutional organization.

\section{Conclusion}

Gene immunization has been going through a lot since its birth. It has very important significance in each stage and made great contributions for the progress of human science and the protection of human life and health. From the existing research results of gene immunization, it still has very broad prospect of future development. But, meanwhile, double efforts shall be made to achieve the target of gene immunization research and to solve the confronting problems of gene immunization research from every perspective provided by the society so as to stimulate the development of gene immunization research. 


\section{References}

[1] Xie Hongye. Recent Progress in Gene Vaccine Research [J]. Chinese Qinghai Journal of Animal and Veterinary Sciences.2004 (02)

[2] Zhu Xinchan, Wang Jinbao. Research Progress of Gene Immunization Vaccine (be continued) [J]. Animal Science and Veterinary Medicine.2002(10)

[3] Sun Changmian, Yang Jiancheng, Zheng Aijuan. Progress in Research of Nucleic Acid Vaccine[J]. Shanghai Journal of Animal Husbandry and Veterinary Medicine.2002(02)

[4] Ma Zhenghai. Research Progress on Enhancing the Immune Effect of DNA Vaccine[J]. Progress in Preventive Veterinary Medicine.1999 (04)

About the Author: Zhang Dandan; gender: female, born in December 1982; native place: Baicheng city, Jilin Province; work unit: College of Life Sciences, Baicheng Normal University; academic title: instructor; research area: immunology; mailing address: Room 502, Unit 3 Building 78, West Zhongxing Road, Baicheng, Jilin

Tel.: 13943663750

Co-corresponding author: Gao Chengguang; gender: male, born in October 1962; native place: Da'an city, Jilin Province; work unit: College of Life Sciences, Baicheng Normal University; academic title: professor; research area: microbiology; mailing address: Room 401, Unit 3 Building 76-1, West Zhongxing Road, Baicheng, Jilin

Tel.: 13843603168 